\title{
State classification for humanoid robots
}

\author{
Jialun Yang ${ }^{\mathrm{a}}$, Feng Gao ${ }^{\mathrm{a}, *}$, Lifeng $\mathrm{Shi}^{\mathrm{a}}$ and Zhenlin $\mathrm{Jin}^{\mathrm{b}}$ \\ ${ }^{a}$ State Key Laboratory of Mechanical System and Vibration, Shanghai Jiao Tong University, Shanghai, 200240, China; ${ }^{b}$ Robotic \\ Research Center, Yanshan University, Qinhuangdao, 066004, China
}

(Received 1 September 2008; final version received 23 January 2009)

\begin{abstract}
In this paper, we decouple the motion-planning problem of humanoid robots into two sub-problems, namely topological state planning and detailed motion planning. The state classification plays a key role for the first sub-problem. We propose several basic states, including lying, sitting, standing and handstanding, abstracted from the daily exercises of human beings. Each basic state is classified further from the topological point of view. Furthermore, generalised function $\left(\mathrm{G}_{\mathrm{F}}\right)$ set theory is applied with the aim of analysing the kinematic characteristics of the end effectors for each state, and meaningful names are assigned for each state. Finally a topological state-planning example is given to show the effectiveness of this methodology. The results show that the large amounts of states can be described using assigned names, which leads to systematic and universal description of the states for humanoid robots.
\end{abstract}

Keywords: state classification; humanoid robots; motion planning.

\section{Introduction}

The research of humanoid robots has drawn more and more attention from researchers all over the world for the last three decades. Along with the fast development of theories and technologies, the capabilities of humanoid robots have grown dramatically (Sakagami et al. 2002; Geppert 2004; Sugahara et al. 2004; Kim et al. 2007). From the development history of humanoid robots, we can see that the era in which human beings and humanoid robots can share the environment simultaneously is approaching, and the tasks for humanoid robots will be more and more complicated.

The motion planning issue is essential for the successful implementation of humanoid robots, especially when the tasks of the humanoid robots are complicated. So far a lot of methodologies have been proposed, and some of them have already been used in humanoid robot platforms. A motioncapturing system (Nakaoka et al. 2003) has been used to acquire human dance motions. The recorded motions are abstracted to get the essential postures in arm motions and step primitives in leg motions. After further procession of the motion data, the humanoid robot is able to imitate the human dances and maintain its balance independently. The movement primitives (Bokman et al. 2005), such as reaching, swinging, lifting light and lifting heavy objects for the forearm, have been represented and stored as a set of joint trajectory basis functions which have been extracted via a principle component analysis of human motion capture data. Through linear combination of those basis functions and optimisation according to some criteria, reasonable in- put data for humanoid robots can be achieved. In the literature (Kuffner et al. 2001), the researchers have employed a randomised search strategy based on rapidly exploring random trees for the planning algorithm in order to generate dynamically stable collision-free trajectories for humanoid robots, and the algorithm has been successfully applied in the H6 humanoid robot. Multiple behavioural primitives (Sentis and Khatib 2005) have been classified into different control categories, i.e. constraints and operational tasks and postures, to achieve safety and efficient control. Figures (Hirukawa et al. 2005) have been used to represent the states of humanoid robot HRP-2P for the process of lying down and getting up. Only typical states are shown, and other intermediate states are omitted to make the description more concise. The humanoid robot HRP-2 (Stilman et al. 2006) interacts with obstacles actively to generate a possible path for the desired task. A general framework (Potkonjak et al. 2006) for the dynamic-modelling issue of human and humanoid motion has been proposed. Moreover, different types of contact between humanoid robots and the environment have also been discussed. A collision-avoidance method (Ohashi et al. 2007) has been described for a biped robot with an upper body. The humanoid robot would stop in front of an obstacle by generating arm force.

From the above introduction in terms of the issue of motion planning for humanoid robots, we can see that the process of solving this issue contains mainly two steps, namely topological state planning (Kuffner et al. 2001; Nakaoka et al. 2003; Bokman et al. 2005; Sentis and Khatib 2005) and

\footnotetext{
*Corresponding author. Email: fengg@sjtu.edu.cn
} 
detailed motion planning (Hirukawa et al. 2005; Potkonjak et al. 2006; Stilman et al. 2006; Ohashi et al. 2007). As a matter of fact, the tasks in previous works (Kuffner et al. 2001; Nakaoka et al. 2003; Bokman et al. 2005; Sentis and Khatib 2005) are performed with such an easy manner that the topological state planning is negligible. In contrast, the tasks described in other works (Hirukawa et al. 2005; Potkonjak et al. 2006; Stilman et al. 2006; Ohashi et al. 2007) are so complicated that topological state planning is mandatory. Along with the further development of humanoid robots, it is straightforward to suggest that topological state planning will become more and more important for the application of humanoid robots. The reason is that in some cases, especially when the tasks for humanoid robots are complicated, humanoid robots should make use of the environment (including movable and unmovable obstacles) and the body of the humanoid robot itself, which leads to many contact states between humanoid robots and the environment. Nevertheless, the problem of topological state planning has been less studied owing to the lack of an appropriate mathematical tool. So the main purpose of this paper is to develop an appropriate mathematical tool for topological state planning, which will be explained in detail later.

The remainder of the paper is organised as follows: The brief introduction of $\mathrm{G}_{\mathrm{F}}$ set theory is given in ' $\mathrm{G}_{\mathrm{F}}$ set theory section'. The humanoid robot platform SJTU-HR1 and its kinematic characteristic tree are covered in a later section. The section 'State classification' presents the categories of basic states of humanoid robots, and the kinematic characteristics of each state are achieved according to $\mathrm{G}_{\mathrm{F}}$ set theory too. The section 'An application example' illustrates the usefulness of this methodology. Finally conclusions appear in the last section.

\section{$\mathbf{G}_{\mathrm{F}}$ set theory}

Normally, the capabilities of robot manipulators contain the translational displacement capability, the rotational displacement capability, the translational velocity capability, the rotational velocity capability, the translational acceleration capability, the rotational acceleration capability and the like, which are linear, numeric, algebraic and variant with respect to coordinate systems and units. On the other hand, the structure of robot manipulators, which has a close relationship with the capabilities of the robot manipulator, is non-linear, non-numeric, non-algebraic and invariant with respect to coordinate systems. Moreover, it is not necessary to use units to describe the structure of robot manipulators. In some cases, robot designers pay more attention to whether the capability exists than to how great the capability is, especially for the type synthesis of robots. So it is useful to define the relationship between the robot structure and the capabilities of robot manipulators.

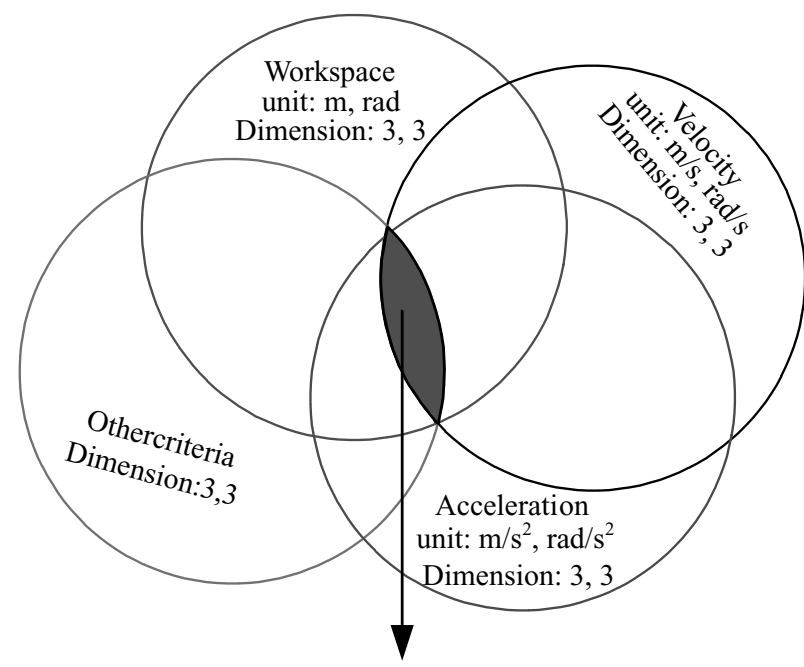

Dimension: 3,3

Figure 1. Common part among capabilities of robot manipulators.

Considering the above-mentioned capabilities (shown in Figure 1) of robot manipulators, we can see that the common part which is the kinematic characteristic of the end effector of robot manipulators is the dimension. Consequently, we employ a special set to describe the kinematic characteristics of the end effector of robot manipulators.

\section{Definition}

The set contains one and only one element which represents the kinematic characteristics of the end effectors of mechanisms and is called the generalised function set $\left(\mathrm{G}_{\mathrm{F}}\right.$ set for short).

Note that the generalised function is the mapping between the specific structure of mechanisms and the kinematic characteristics of the end effectors of mechanisms. And the kinematic characteristics of the end effector of mechanisms can be described using a $\mathrm{G}_{\mathrm{F}}$ set with the form of $\left(T_{a} T_{b} T_{c} ; R_{\alpha} R_{\beta} R_{\gamma}\right)$, where $T_{a} T_{b} T_{c}$ denote translational displacements of the end effector about vectors $a, b$ and $c,(a \times b) \cdot c \neq 0$, and $R_{\alpha} R_{\beta} R_{\gamma}$ describe the rotational displacements of the end-effector with respect to three Euler's angles $\alpha, \beta$ and $\gamma$. Note that the vectors $T_{a} T_{b} T_{c}$ are non-co-planar simultaneously, and the vectors $R_{\alpha} R_{\beta} R_{\gamma}$ always intersect at a common point and are non-co-planar simultaneously. The non-zero components of the $\mathrm{G}_{\mathrm{F}}$ set represent the existence of the corresponding capabilities of the end effector. For instance, the $\operatorname{set}\left(T_{a} T_{b} T_{c} ; 0000\right)$ means that three translation displacements exist, and the set ( $\begin{array}{llll}0 & 0 & 0\end{array} R_{\alpha} R_{\beta} R_{\gamma}$ ) indicates three rotational displacements exist. The $\mathrm{G}_{\mathrm{F}}$ set theory (Gao, Li et al. 2002; Gao, Zhang et al. 2005) has been applied to the synthesis of novel parallel mechanisms. Furthermore, the 
$\mathrm{G}_{\mathrm{F}}$ set theory (Yu et al. 2008) has also been employed to generate several new structures of forging manipulators.

In the following subsections, we will enrich the $G_{\mathrm{F}}$ set theory in terms of its operation rules and application field.

The operation rules of $\mathrm{G}_{\mathrm{F}}$ sets are shown next.

\section{Union operation of $G_{F}$ sets}

Let $\mathrm{G}_{F 1}, \mathrm{G}_{F 2}, \ldots, \mathrm{G}_{F N}$ represent $N G_{F}$ sets. The union of those $G_{F}$ sets can be expressed as Equation (1):

$$
G_{F}=G_{F 1} \cup G_{F 2} \ldots \cup G_{F N} .
$$

\section{Intersection operation of $G_{\mathrm{F}}$ sets}

Let $G_{F 1}, G_{F 2}, \ldots, G_{F N}$ represent $N G_{F}$ sets. The intersection of those $\mathrm{G}_{F}$ sets is as in Equation (2):

$$
G_{F}=G_{F 1} \cap G_{F 2} \ldots \cap G_{F N} .
$$

\section{Reversion operation of $G_{F}$ sets}

The reversion operation of $\mathrm{G}_{F}$ sets is shown in Equation (3):

$$
\left(G_{F}\right)^{R}=G_{F}^{R}
$$

\section{Reversion operation of the union of $G_{F}$ sets}

The reversion operation of the union of $G_{F}$ sets can be computed as Equation (4):

$$
\begin{aligned}
& \left(G_{F 1} \cup G_{F 2} \cup \ldots \cup G_{F N}\right)^{R} \\
& \quad=G_{F N}^{R} \cup G_{F(N-1)}^{R} \cup \ldots \cup G_{F 2}^{R} \cup G_{F 1}^{R}
\end{aligned}
$$

The theorems regarding the application of the $\mathrm{G}_{F}$ set theory are as follows.

\section{Theorem for serial mechanisms}

For a serial mechanism, the kinematic characteristics of the end effector of the mechanism are the union of all of the $\mathrm{G}_{F}$ sets with such a sequence that from the base of the mechanism to the end effector. For instance, the kinematic characteristics of the third robot manipulator composed of the first and the second robot manipulators (see Figure 2) are $G_{F 1} \cup G_{F 2}$, which is the union of the $\mathrm{G}_{F}$ sets of each kinematic chain.

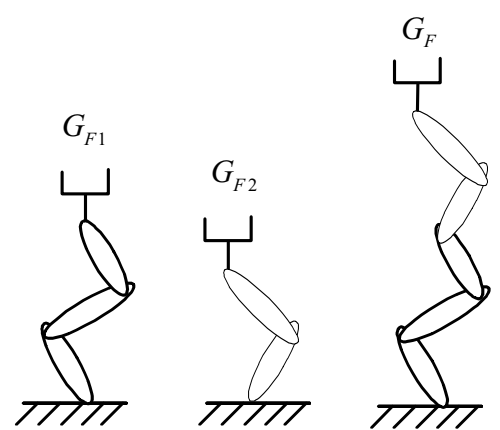

Figure 2. Kinematic characteristics of serial mechanisms.

\section{Theorem for parallel mechanisms}

The kinematic characteristics of the moving platform of a parallel mechanism are the intersection of the $\mathrm{G}_{F}$ sets of each kinematic chain of the parallel mechanism. For example, the kinematic characteristics of the moving platform of the parallel mechanism (see Figure 3) are $G_{F 1} \cap G_{F 2} \cap G_{F 3}$, which is the intersection of $\mathrm{G}_{F}$ sets of each kinematic chain.

The application of the reversion operation is related to the mechanism whose end effector and base are exchanged. It is worth noting that the sequence of the axes of the mechanism affects the kinematic characteristics of the end effectors of the mechanism. So we have to pay due attention to the sequence of the $\mathrm{G}_{F}$ sets to achieve correct kinematic characteristics of the end effectors of mechanisms.

\section{Humanoid robot platform and its characteristics tree}

The human skeleton contains 206 bones and has more than 300 degrees of freedom (DOFs) driven by more than 600 muscles. Generally, humanoid robots need not feature as many DOFs as the human skeleton. From the biological point of view, we have been developing a humanoid robot platform SJTU-HR1, whose computer-aided design (CAD) model is shown in Figure 4(a). There exist three types of joints in SJTU-HR1 according to the number of DOFs the joints have. For example, the shoulder, waist and hip joints

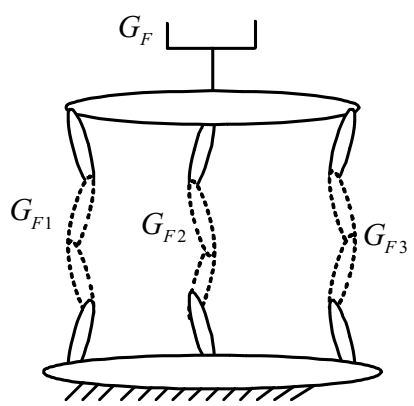

Figure 3. Kinematic characteristics of parallel mechanisms. 


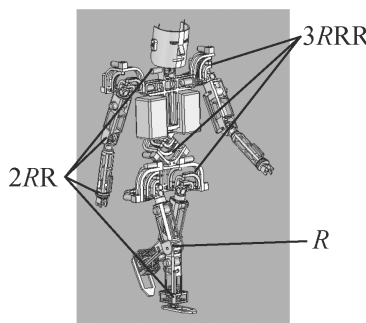

(a)

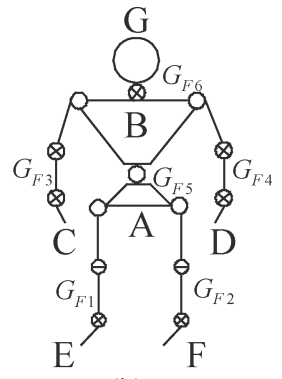

(b)
Figure 4. (a) Three-dimensional model of SJTU-HR1; (b) characteristics tree of SJTU-HR1.

feature three DOFs, with a structure of a $3 \underline{R R R}$ spherical parallel mechanism which is the same as the mechanism of the agile eye (Gosselin et al. 1996). The neck, elbow, wrist and ankle joints have two DOFs, with a structure of an orthogonal spherical parallel mechanism which is the same as the spherical 5R mechanism (Cervantes-Sanchez et al. 2004). The knee joints feature one DOF, with a structure of revolute joints. Most of the motors can be mounted on or near the base, thanks to the introduction of parallel mechanisms. Moreover, multiple joints having the same structure could lead to modularity design and manufacture for the SJTU-HR1. As a result, the SJTU-HR1 enjoys the advantage of both parallel mechanisms and serial mechanisms.

Figure 2(b) is the characteristics tree of SJTU-HR1, which is the sketch of SJTU-HR1 represented by $\mathrm{G}_{F}$ sets. Particularly, in Figure 4(b), the empty small circle represents the joints featuring three DOFs; the small circle with cross indicates the joints with two DOFs; and the small circle with bar depicts the joints with one DOF. Moreover, the letters A, B, C, D, E, F and G in Figure 4(b) represent pelvis, thorax, right hand, left hand, right foot, left foot and head, respectively. Since the sequence of the axes of the robot affects the kinematic characteristics of the end effectors, we let the $G_{F}$ sets from A to E, F, B and the $G_{F}$ sets from B to C, D, G be $G_{F 1}, G_{F 2}, G_{F 3}, G_{F 4}, G_{F 5}$ and $G_{F 6}$, respectively. Making use of the characteristics tree of SJTU-HR1, we can achieve the kinematic characteristics of the interested end effectors of SJTU-HR1 for specific state.

\section{State classification}

\section{Contacts between robots and the environment}

Basically, several types of contact exist when humanoid robots interact with the environment(Potkonjak et al. 2006). Figure 5 depicts four different contacts, namely point contact [Figure 5(a)], line contact [Figure 5(b)], free contact [Figure 5(c)] and fixed contact [Figure 5(d)]. Particularly, the foot shown in Figure 5(a) can rotate about the contact point; the foot shown in Figure 5(b) has one rotation around the line between the foot and the environment; the foot shown in Figure 5(c) could move freely with respect

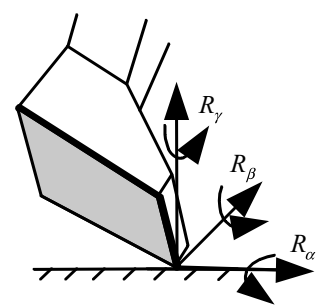

(a) Point contact

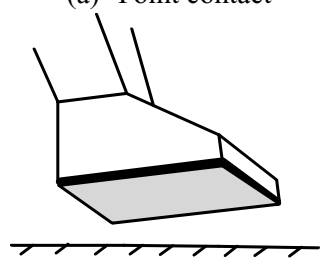

(c) Free contact

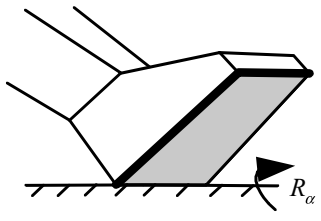

(b) Line contact

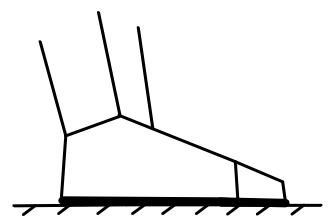

(d) Fixed contact

Figure 5. Sketches for several contacts.

to the environment; the foot shown in Figure 5(d) is fixed to the environment, and there is no relative displacement between the shoe and the environment. Hereafter we will use Full $\mathrm{G}_{F}$ set to represent the contact of Figure 5(c) and Null $G_{F}$ set to indicate the contact of Figure 5(d).

As a matter of fact, other kinds of contact exist too; for instance the hand of a humanoid robot moves on one plane; the foot of a humanoid robot skates on the ice; and so on. However, we only take the fixed contact into account, which means that as soon as the link contacts the environment, no relative motions exist between the link and the environment.

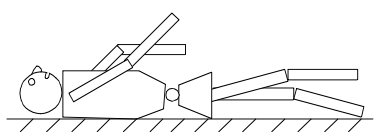

(a)

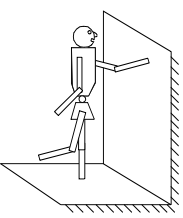

(c)

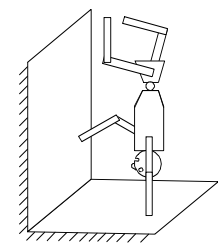

(f)

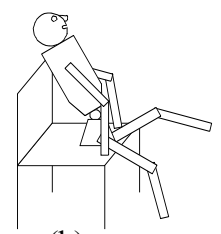

(b)

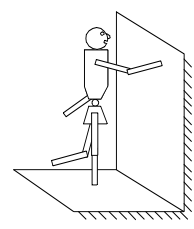

(d)

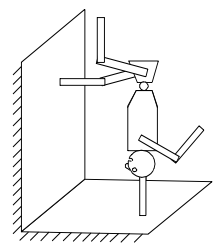

(g)

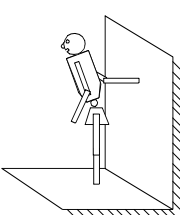

(e)

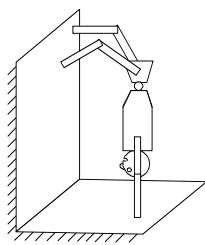

(h)

Figure 6. Basic states of SJTU-HR1. 


\section{Basic states for the humanoid robot platform SJTU-HRI}

From Figure 4(b), we can see that the SJTU-HR1 consists of 15 links connected by 14 joints. Theoretically speaking, all of the 15 links of SJTU-HR1 can function as end effectors which are able to interact with the environment. The number of both the links having contact with the environment and the links connected to the environment refer to different states of humanoid robots (see Figure 6). As a result, the total number of the states could be computed as in Equation (5):

$$
N=C_{15}^{1}+C_{15}^{2}+\ldots+C_{15}^{15}=32767,
$$

where $N$ is the total number of the states and $C_{15}^{i}, i=$ $1, \ldots, 15$, is the number of choices of choosing $i$ links as the end effectors of the humanoid robot from 15 links.

The result in Equation (5) is quite large even without taking the self-connection cases into account. Apparently, it is necessary and meaningful to abstract some general and commonly used states from the daily exercise of human beings, which will be applied to humanoid robots. Consequently, we propose several states as basic states, including lying, sitting, standing and handstanding (Figure 6).

Within each basic state, multiple states exist, and we have to classify them systematically. As a result, the concept of primary support and secondary support for each basic state is proposed. In particular, the primary support is the common support that basic states should feature, and the secondary support can be used for further classifying the basic states. So the primary support for each basic state is different. The secondary support for each basic state can be found in Table 1, and the number of states of each kind of basic state is computed from the topological point of view.

Obviously, the primary support of each state in Figures 6(a)-(h) are thorax and pelvis, pelvis, left foot, right foot, both feet, left hand, right hand and both hands, respectively. Moreover, the secondary support of those state in Figures 6(a)-(h) are left foot, right foot and both hands, left hand, right hand, right hand, left hand, left foot and both feet, respectively.

\section{Kinematic characteristics of lying states}

As indicated in Table 1, 16 lying states exist, and three of them are listed in Table 2, including the corresponding kinematic characteristics for each interested end effector as well as the name for each state. The kinematic characteristics of the interested end effectors are achieved via the characteristics tree of humanoid robot platform SJTU-HR1 and the $G_{F}$ set theory. Note that other interested end effectors featuring a Null $G_{F}$ set are not listed in Table 2, as well as Tables 3-5, for the sake of conciseness.
Table 1. Basic state classification for humanoid robots.

\begin{tabular}{lllc}
\hline Basic state & $\begin{array}{c}\text { Primary } \\
\text { support }\end{array}$ & $\begin{array}{l}\text { Possible secondary } \\
\text { support }\end{array}$ & $\begin{array}{c}\text { Number of } \\
\text { states }\end{array}$ \\
\hline Lying & $\begin{array}{l}\text { Pelvis } \\
\text { Thorax } \\
\text { Peltinis }\end{array}$ & Four limbs & 16 \\
Standing & Left foot & $\begin{array}{l}\text { Four limbs } \\
\text { Thorax } \\
\text { Pelvis } \\
\text { Right foot } \\
\text { Both hands } \\
\text { Pelvis } \\
\text { Left foot } \\
\text { Both hands } \\
\text { Handstanding }\end{array}$ & Left hand \\
Both feet & $\begin{array}{l}\text { Both hands } \\
\text { Pelvis } \\
\text { Both feet } \\
\text { Right hand } \\
\text { Pelvis } \\
\text { Both feet } \\
\text { Left hand } \\
\text { Pelvis } \\
\text { Both feet } \\
\text { Pelvis }\end{array}$ & 16 \\
\hline Right hand & 16 \\
\hline & Both hands & 16 \\
\hline
\end{tabular}

Table 2. Lying states.

\begin{tabular}{lllc}
\hline Name & $\begin{array}{l}\text { Secondary } \\
\text { support }\end{array}$ & $\begin{array}{l}\text { Interested } \\
\text { end effectors }\end{array}$ & $\begin{array}{c}\text { Kinematic } \\
\text { characteristics }\end{array}$ \\
\hline$L_{f l}$ & Right foot & Right foot & $G_{F 1}$ \\
& & $\begin{array}{l}\text { Right hand } \\
\text { Left hand }\end{array}$ & $G_{F 3}$ \\
& & Head & $G_{F 4}$ \\
$L_{h r h l}$ & Right hand Left hand & Right foot & $G_{F 6}$ \\
& & Left foot & $G_{F 2}$ \\
$L_{A}$ & Both feet & Head & $G_{F 6}$ \\
& Both hands & Head & $G_{F 6}$ \\
& & & \\
\hline
\end{tabular}

Table 3. Sitting states.

\begin{tabular}{|c|c|c|c|}
\hline Name & $\begin{array}{l}\text { Secondary } \\
\text { support }\end{array}$ & $\begin{array}{l}\text { Interested } \\
\text { end effectors }\end{array}$ & $\begin{array}{l}\text { Kinematic } \\
\text { characteristics }\end{array}$ \\
\hline$S_{f r}$ & Right foot & $\begin{array}{l}\text { Left foot } \\
\text { Right hand } \\
\text { Left hand } \\
\text { Head } \\
\text { Thorax }\end{array}$ & $\begin{array}{l}G_{F 2} \\
G_{F 5} \cup G_{F 3} \\
G_{F 5} \cup G_{F 4} \\
G_{F 5} \cup G_{F 6} \\
G_{F 5}\end{array}$ \\
\hline$S_{f l t h}$ & Left foot Thorax & $\begin{array}{l}\text { Right foot } \\
\text { Right hand } \\
\text { Left hand } \\
\text { Head }\end{array}$ & $\begin{array}{l}G_{F 1} \\
G_{F 3} \\
G_{F 4} \\
G_{F 6}\end{array}$ \\
\hline$S_{F R}$ & $\begin{array}{l}\text { Left foot } \\
\text { Both hands } \\
\text { Thorax }\end{array}$ & $\begin{array}{l}\text { Right foot } \\
\text { Head }\end{array}$ & $\begin{array}{l}G_{F 1} \\
G_{F 6}\end{array}$ \\
\hline
\end{tabular}


Table 4. Standing states.

\begin{tabular}{|c|c|c|c|}
\hline Name & $\begin{array}{l}\text { Secondary } \\
\text { support }\end{array}$ & $\begin{array}{l}\text { Interested } \\
\text { end effectors }\end{array}$ & Kinematic characteristics \\
\hline$S B_{N}$ & & $\begin{array}{l}\text { Right hand } \\
\text { Left hand } \\
\text { Head } \\
\text { Thorax } \\
\text { Pelvis }\end{array}$ & $\begin{array}{l}\left(G_{F 1}^{R} \cap G_{F 2}^{R}\right) \cup G_{F 5} \cup G_{F 3} \\
\left(G_{F 1}^{R} \cap G_{F 2}^{R}\right) \cup G_{F 5} \cup G_{F 4} \\
\left(G_{F 1}^{R} \cap G_{F 2}^{R}\right) \cup G_{F 5} \cup G_{F 6} \\
\left(G_{F 1}^{R} \cap G_{F 2}^{R}\right) \cup G_{F 5} \\
G_{F 1}^{R} \cap G_{F 2}^{R}\end{array}$ \\
\hline$S B_{P E}$ & Right hand Left hand & $\begin{array}{l}\text { Head } \\
\text { Thorax } \\
\text { Pelvis }\end{array}$ & $\begin{array}{l}\left(\left(\left(G_{F 1}^{R} \cap G_{F 2}^{R}\right) \cup G_{F 5}\right) \cap G_{F 3}^{R} \cap G_{F 4}^{R}\right) \cup G_{F 6} \\
\left(\left(G_{F 1}^{R} \cap G_{F 2}^{R}\right) \cup G_{F 5}\right) \cap G_{F 3}^{R} \cap G_{F 4}^{R} \\
G_{F 1}^{R} \cap G_{F 2}^{R} \cap\left(\left(G_{F 3}^{R} \cap G_{F 4}^{R}\right) \cup G_{F 5}^{R}\right)\end{array}$ \\
\hline$S B_{h l}$ & Left hand & $\begin{array}{l}\text { Right hand } \\
\text { Head } \\
\text { Thorax } \\
\text { Pelvis }\end{array}$ & $\begin{array}{l}\left(\left(\left(G_{F 1}^{R} \cap G_{F 2}^{R}\right) \cup G_{F 5}\right) \cap G_{F 4}^{R}\right) \cup G_{F 3} \\
\left(\left(\left(G_{F 1}^{R} \cap G_{F 2}^{R}\right) \cup G_{F 5}\right) \cap G_{F 4}^{R}\right) \cup G_{F 6} \\
\left(\left(G_{F 1}^{R} \cap G_{F 2}^{R}\right) \cup G_{F 5}\right) \cap G_{F 4}^{R} \\
G_{F 1}^{R} \cap G_{F 2}^{R} \cap\left(G_{F 4}^{R} \cup G_{F 5}^{R}\right)\end{array}$ \\
\hline$S L_{A}$ & $\begin{array}{l}\text { Right foot } \\
\text { Both hands } \\
\text { Pelvis }\end{array}$ & $\begin{array}{l}\text { Head } \\
\text { Thorax }\end{array}$ & $\begin{array}{l}\left(G_{F 3}^{R} \cap G_{F 4}^{R} \cap G_{F 5}\right) \cup G_{F 6} \\
G_{F 3}^{R} \cap G_{F 4}^{R} \cap G_{F 5}\end{array}$ \\
\hline$S R_{H L}$ & $\begin{array}{l}\text { Left foot } \\
\text { Right hand } \\
\text { Left hand }\end{array}$ & $\begin{array}{l}\text { Left hand } \\
\text { Head } \\
\text { Thorax }\end{array}$ & $\begin{array}{l}\left(G_{F 3}^{R} \cap G_{F 5}\right) \cup G_{F 4} \\
\left(G_{F 3}^{R} \cap G_{F 5}\right) \cup G_{F 6} \\
G_{F 3}^{R} \cap G_{F 5}\end{array}$ \\
\hline
\end{tabular}

The name of each state is given following such a rule that the term ' $\mathrm{L}$ ' denotes lying, and all of other terms in the subscript indicate different secondary supports. It is not difficult to see that the name of each state can represent the specified state. Furthermore, more information regarding the capability of this state can be achieved considering the kinematic characteristics of the related end effectors of each state.

Kinematic characteristics of sitting, standing and handstanding states

Tables 3-5 list several sitting, standing and handstanding states, respectively. Similar to the name of lying states, the terms 'S', 'SL', 'SR', 'SB', 'HL', 'HR', 'HB', denote the

Table 5. Handstanding states.

\begin{tabular}{lcll}
\hline Name & $\begin{array}{c}\text { Secondary } \\
\text { support }\end{array}$ & $\begin{array}{l}\text { Interested } \\
\text { end effectors }\end{array}$ & $\begin{array}{l}\text { Kinematic } \\
\text { characteristics }\end{array}$ \\
\hline$H L_{h r}$ & Right hand & Right foot & $\left(G_{F 3}^{R} \cap G_{F 4}^{R}\right) \cup G_{F 5}^{R} \cup G_{F 1}$ \\
& & Left foot & $\left(G_{F 3}^{R} \cap G_{F 4}^{R}\right) \cup G_{F 5}^{R} \cup G_{F 2}$ \\
& & Head & $\left(G_{F 3}^{R} \cap G_{F 4}^{R}\right) \cup G_{F 6}$ \\
& & Thorax & $G_{F 3}^{R} \cap G_{F 4}^{R}$ \\
& & pelvis & $\left(G_{F 3}^{R} \cap G_{F 4}^{R}\right) \cup G_{F 5}^{R}$ \\
$H R_{f r}$ & Right foot & Left hand & $\left(G_{F 3}^{R} \cap\left(G_{F 1}^{R} \cup G_{F 5}\right)\right) \cup G_{F 4}$ \\
& & Left foot & $\left(\left(G_{F 3}^{R} \cup G_{F 5}^{R}\right) \cap G_{F 1}^{R}\right) \cup G_{F 2}$ \\
& & Head & $\left(G_{F 3}^{R} \cap\left(G_{F 1}^{R} \cup G_{F 6}\right.\right.$ \\
& & Thorax & $G_{F 3}^{R} \cap\left(G_{F 1}^{R} \cup G_{F 5}\right)$ \\
$H B_{A}$ & Head & Pelvis & $\left(G_{F 3}^{R} \cup G_{F 5}^{R}\right) \cap G_{F 1}^{R}$ \\
& Pead & $\left(G_{F 3}^{R} \cap G_{F 4}^{R} \cap G_{F 5}\right) \cup G_{F 6}$ \\
& Pelvis & Pelvis & $G_{F 3}^{R} \cap G_{F 4}^{R} \cap G_{F 5}$ \\
\hline
\end{tabular}

sitting, standing with left foot, standing with right foot, standing with both feet, handstanding with left hand, handstanding with right hand and handstanding with both hands, respectively.

Again, we can make use of the names to represent the specified states, instead of using pictures. And such representation can show sufficiently the relationship between the kinematic characteristics of the interested end effectors of the SJTU-HR1 and the related state.

\section{An application example}

The process for the SJTU-HR1 from lying on the ground to getting up is employed to illustrate the application of the methodology of this paper. Multi-state paths for this process are illustrated in Figure 7, from which we can see that all of the possible lying states, sitting states and standing

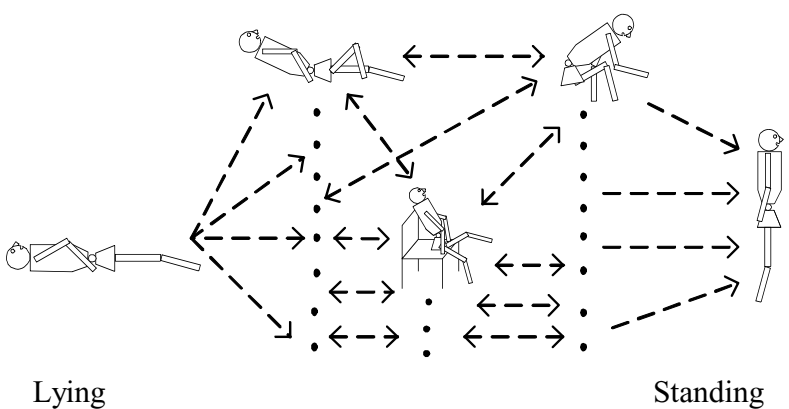

Figure 7. Multi-state paths for the process from lying on the ground to getting up. 


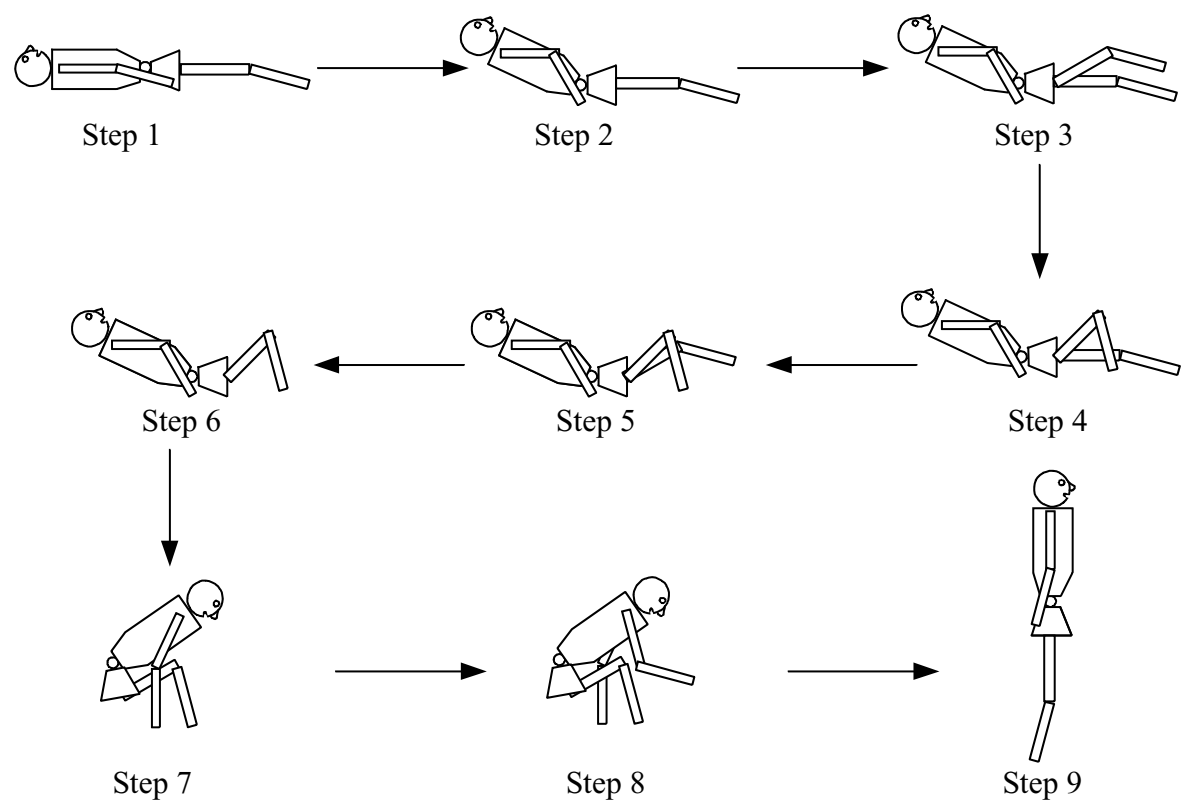

Figure 8. Sketches of the process from lying on the ground to getting up.

states can function as intermediate states, which leads to the complexity of the state-planning issue

Figure 8 depicts one optimal path achieved according to the criterion of maximum contact points. The fact is that the more contact points, the easier it is for the robot to get up. That's why for the step 2, the humanoid robot SJTUHR1 makes use of two hands to support its body instead of only one hand. However, the understandability of Figure 8 depends highly on the skill of painters. Moreover, some misunderstandings are possible, given that the quality of the graph is not high.

Unlike Figure 8, we use the names achieved via the $G_{F}$ set theory to describe the process from lying on the ground to getting up (Figure 9). Each name has its own meaning in terms of the kinematic characteristics of the interested end effectors of SJTU-HR1 and is able to represent the related state. Obviously, the expression in Figure 9 is standard, and it can be interpreted universally without any confusion. Fur-

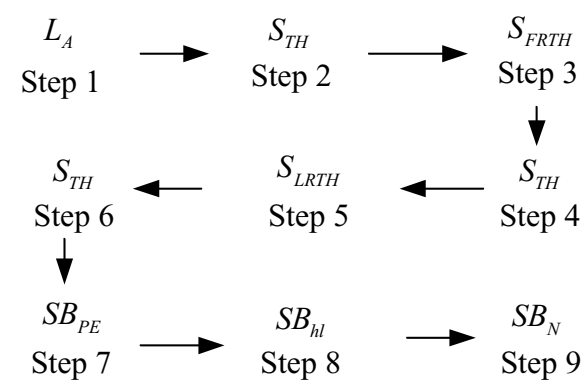

Figure 9. Description of the process from lying on the ground to getting up, using names. thermore, this methodology can be used for the topological state planning, taking some optimal criteria into account, since multiple state paths exist for one given task.

\section{Conclusion}

This work focuses on state classification for humanoid robots, in particular the humanoid platform SJTU-HR1. The main contributions of our work are as follows:

- several basic states are proposed according to the concept of primary support, such as the states of lying, sitting, standing and handstanding;

- the characteristics tree of the SJTU-HR1 is established;

- the kinematic characteristics of the interested end effectors of the SJTU-HR1 are achieved making use of $\mathrm{G}_{F}$ set theory; and

- one application example is given to show that the results achieved via the methodology proposed in this paper are more concise, systematic and informative.

The work presented in this paper concerns the state classification for the SJTU-HR1. However, the authors believe that this methodology can also be applied for analysing other types of robots, such as quadruped robots and hexapedal robots.

\section{Acknowledgment}

This work was support by Natural Basic Research Program of China (grant no. 2006CB705402) and partially by National 
Natural Science Foundation of China (grant no. 60534020) and the Graduate Innovation Foundation of Shanghai Jiao Tong University. We would also like to thank the anonymous reviewers for their very useful comments.

\section{References}

Bokman L, Syungkwon R, Park FC. 2005. Movement primitives, principal component analysis, and the efficient generation of natural motions. In: Proceedings of the 2005 IEEE International Conference on Robotics and Automation, IEEE, Barcelona, Spain, p. 4630-4635.

Cervantes-Sanchez JJ, Hernandez-Rodriguez JC, GonzalezGalvan EJ. 2004. On the 5R spherical, symmetric manipulator: workspace and singularity characterization. Mechan Machine Theory 39(4):409-429.

Gao F, Li W, Zhao X, Jin Z, Zhao H. 2002. New kinematic structures for 2-, 3-, 4-, and 5-DOF parallel manipulator designs. Mechan Machine Theory 37(11):1395-1411.

Gao F, Zhang Y, Li W. 2005. Type synthesis of 3-DOF reducible translational mechanisms. Robotica. 23(2):239-245.

Geppert L. 2004. QRIO the robot that could. IEEE Spect. 41(5):34-36.

Gosselin CM, St. Pierre E, Gagne M. 1996. On the development of the agile eye. IEEE Rob Automat Mag. 3(4):29-37.

Hirukawa H, Kajita S, Kanehiro F, Kaneko K, Isozumi T. 2005. The human-size humanoid robot that can walk, lie down and get up. Int J Rob Res. 24(9):755-769.

Kim JY, Lee J, Oh JH. 2007. Experimental realization of dynamic walking for a human-riding biped robot, hubofx-1. Adv Rob. 21(3-4):461-484.

Kuffner J, Nishiwaki K, Kagami S, Inaba M, Inoue H. 2001. Motion planning for humanoid robots under obstacle and dy- namic balance constraints. Seoul: Institute of Electrical and Electronics Engineers Inc., 692-698.

Nakaoka S, Nakazawa A, Yokoi K, Hirukawa H, Ikeuchi K. 2003. Generating whole body motions for a biped humanoid robot from captured human dances. 3:3905-3910.

Ohashi E, Aiko T, Tsuji T, Nishi H, Ohnishi K. 2007. Collision avoidance method of humanoid robot with arm force. IEEE Trans Indus Electron. 54(3):1632-1641.

Potkonjak V, Vukobratovic M, Babkovic K, Borovac B. 2006. General model of dynamics of human and humanoid motion: Feasibility, potentials and verification. Int J Humanoid Rob. 3(1):21-47.

Sakagami Y, Watanabe R, Aoyama C, Matsunaga S, Higaki N, Fujimura K. 2002. The intelligent ASIMO: system overview and integration. In: Proceedings of the 2002 IEEE/RSJ International Conference on Intelligent Robots and Systems, vols. $1-3$, , p. 2478-2483.

Sentis L, Khatib O. 2005. Synthesis of whole-body behaviors through hierarchical control of behavioral primitives. Int $\mathbf{J}$ Humanoid Rob. 2(4):505 - 518.

Stilman M, Nishiwaki K, Kagami S, Kuffner JJ. 2006. Planning and executing navigation among movable obstacles. In: 2006 IEEE/RSJ International Conference on Intelligent Robots and Systems. p. 820-826.

Sugahara Y, Hosobata T, Mikuriya Y, Sunazuka H, Hun-Ok L, Takanishi A. 2004. Realization of dynamic human-carrying walking by a biped locomotor. In: 2004 IEEE International Conference on Robotics and Automation. New Orleans, LA: IEEE . p. 3060-3065.

Yu F, Gao F, Shi Q, Ge H, Zhang Y. 2008. Structure synthesis for forging manipulators. In: WCICA 2008. 7th World Congress on Intelligent Control and Automation. p. 400403. 

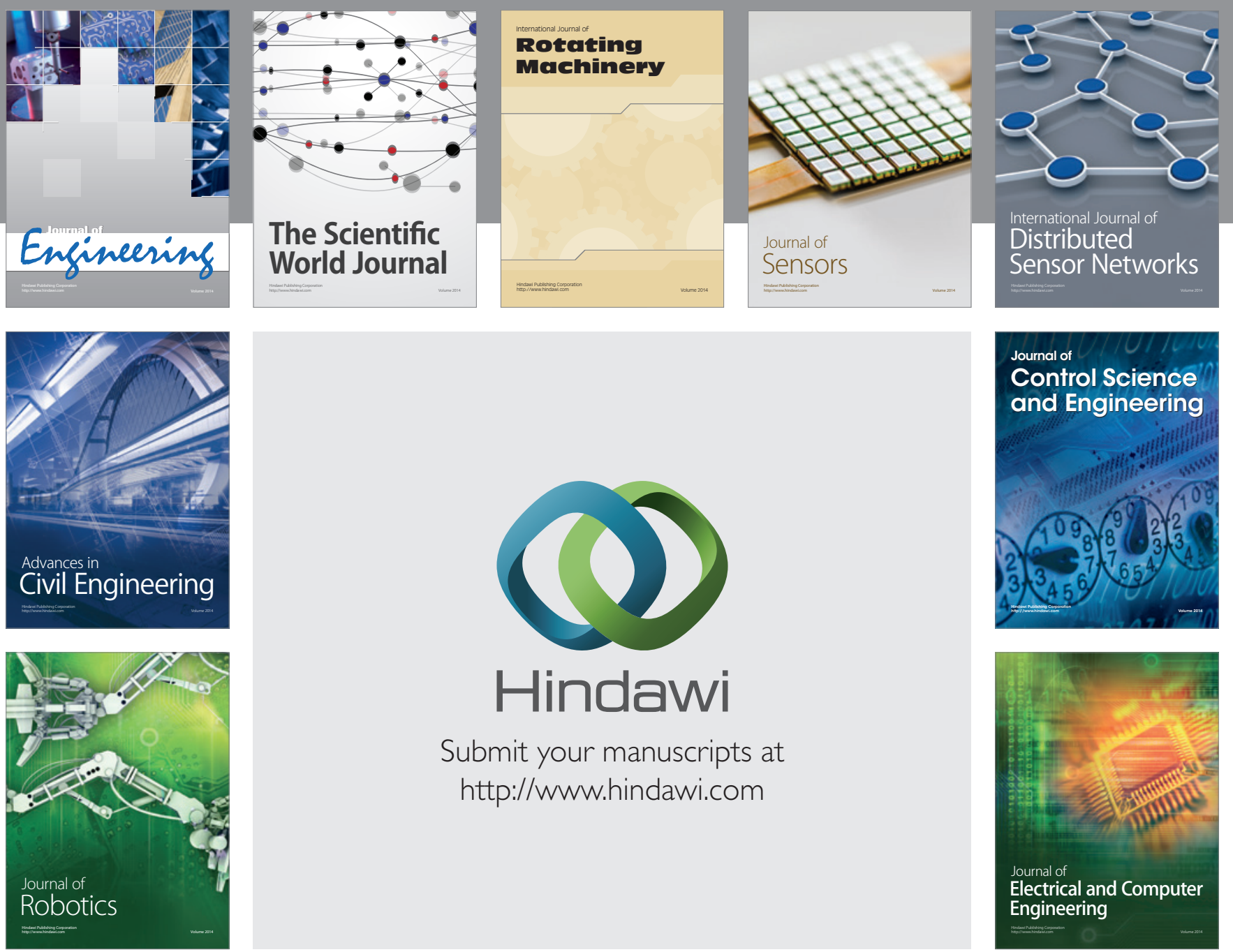

Submit your manuscripts at

http://www.hindawi.com
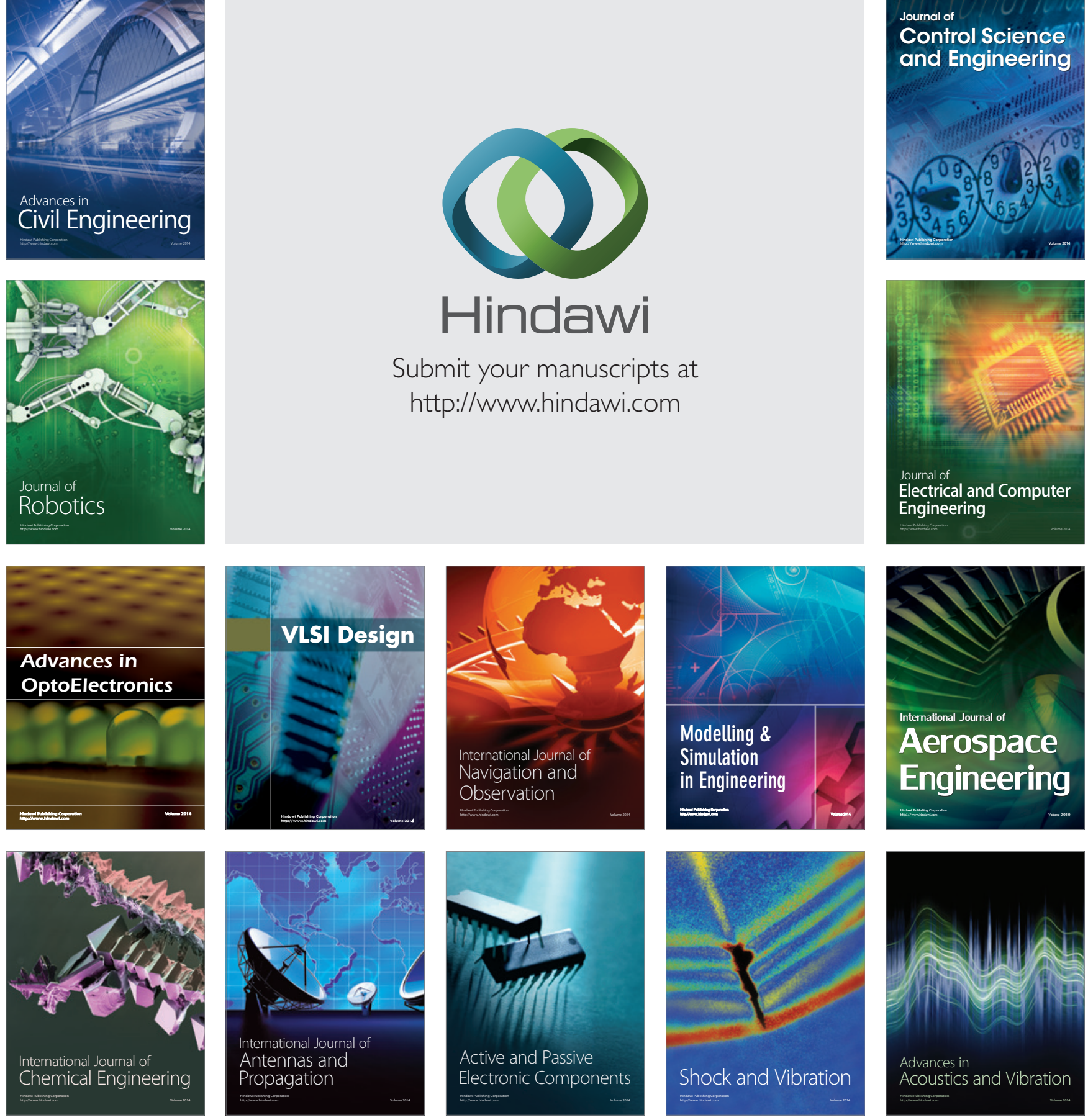